\title{
Ni ciudadanos ni extranjeros: los itálicos en la política de los tribunos de la plebe a principios de la crisis de la República romana
}

\author{
Neither citizens nor foreigners: the Italics in the politics of the \\ tribuni plebis in the begining of the crisis of the Roman Republic
}

\author{
LUIS MANUEL LÓPEZ ROMÁN*
}

RESUMEN

Desde la extensión del dominio romano más allá del Lacio, el modelo de relación entre

Roma y las diversas comunidades itálicas se basó, por un lado, en la autonomía interna de éstas y, por otro, en la concesión de una serie de ventajas como compensación por los servicios prestados a la Urbe y por la limitación de su soberanía. Sin embargo, la expansión de las armas romanas por el Mediterráneo y la política de imperialismo agresivo llevarán a Roma a aumentar las exigencias hacia las comunidades itálicas, sin concederles, como contrapartida, una mayor integración en el sistema de gobierno del Estado. Debido a esto, la cuestión de los itálicos se convertirá desde inicios de la crisis de la República en un tema de preocupación constante para los políticos romanos. El objetivo de esta comunicación es el análisis de las políticas llevadas a cabo por los tribunos de la plebe relacionadas con la integración de los itálicos desde el año 133 a.C. hasta la extensión de la ciudadanía romana completa a todos los socii a finales de los años 90.

\begin{abstract}
Since the Romans extended their domination beyond the Latium, the Urbs established with the Italic communities a model of relationship based on the internal autonomy and the concession of several advantages for them. However, during the expansion of the Roman supremacy throughout the Mediterranean, Rome increased her demands to the Italics, not granting them in exchange a better form of integration in the state. Because of that, the Italic issue will be, from the begining of the crisis of the Republic, a cause of constant worry for Roman politicians. The main point of this paper is the analysis of the politics related to the integration of the Italics carried out by the tribuns of the plebs from the year 133 B.C. until the concession of the Roman citizenship to all the socii at the end of the 90's.
\end{abstract}

* Departamento de Historia Antigua, Universidad Complutense de Madrid (C/ Profesor Aranguren, s/n. 28040 Madrid). E-mail: pompeyo82@ hotmail.com.

Artículo basado en la comunicación leída el 25 de Mayo de 2009, en la VIII edición del Encuentro de Jóvenes Investigadores de Historia Antigua de la Universidad Complutense de Madrid. 
PALABRAS CLAVE:

Itálicos, ciudadanía, tribunado de la plebe, socii.
KEYWORDS:

Italics, citizenship, tribunate of the plebs, socii.

\section{EL TRIBUNADO DE LA PLEBE Y LA CUESTIÓN DE LOS ITÁLICOS}

La cuestión de los itálicos y su integración en el sistema político romano es un fenómeno que ha llamado singularmente la atención a los historiadores del mundo antiguo ya desde el siglo XIX, y que, sin embargo, por sus especiales características y por las conexiones que a menudo han querido establecerse entre este proceso y otros propios de la modernidad, ha recibido interpretaciones variadas e incluso contrapuestas. El desarrollo de la historiografía en paralelo al de las corrientes nacionalistas de algunos países europeos, especialmente Italia, llevó a los estudiosos a ver semejanzas entre el dominio que ejerció Roma sobre sus aliados peninsulares y la opresión de Europa por parte de Napoleón y sus ejércitos (Mascioli 1942). Por el contrario, el proceso de unificación alemana condicionó sin duda a Mommsen a la hora de elaborar la interpretación que más peso e influencia ejerció en la historiografía posterior. Según este autor, Roma y los latinos habrían sido el motor de una beneficiosa unificación italiana, de tipo político y lingüístico, que sólo comenzó a resquebrajarse en el siglo Il a.C (Mommsen 1888). Encontramos, por tanto, una férrea distinción entre un idílico siglo III a.C. y un siglo II a.C. en el que las perfidias constantes de determinados políticos romanos llevarán a los itálicos al descontento, en primer lugar, y a la rebelión abierta en última instancia.

Esta visión de una Roma paternalista y unos aliados deseosos de ser romanizados se aceptó durante buena parte del siglo XX sin apenas críticas, lo que convirtió en verdades absolutas cuestiones que, según se demostró posteriormente, apenas encuentran un apoyo documental en las fuentes. Sólo en las últimas décadas se ha procedido a una revisión crítica de estos postulados, hecho éste que ha permitido, mediante una lectura más rigurosa y menos condicionada de los textos antiguos, descubrir que numerosas afirmaciones antes incontestadas eran en realidad bases erróneas sobre las cuales no podía construirse ningún tipo de argumentación. Sin duda, es el trabajo del profesor Wulff Alonso el que más contribuciones de valor ha realizado en este sentido, ya que rompió la tendencia a seguir la tradición de Mommsen de una manera acrítica y presentó nuevas bases para abordar la cuestión de los itálicos desde una perspectiva más sólida (Wulff 1991).

Por otro lado, la interpretación tradicional de la cuestión itálica ha ejercido durante décadas una notable influencia en los estudios sobre otros aspectos políticos internos de la República romana. Esto se debe a que, al menos desde mediados del siglo II a.C., el aumento de la presión y de las exigencias por parte de Roma sobre sus aliados peninsulares y la negativa de la aristocracia senatorial a conceder a cambio una mayor capacidad de intervención en la política de la Urbe 
Ni ciudadanos ni extranjeros: los itálicos en la política de los tribunos de la plebe...

llevó a los itálicos a una situación de indudable malestar y descontento cuyos ecos se manifestaron con fuerza creciente en Roma. El factor de los aliados comenzó entonces a ser una cuestión preocupante que algunos políticos romanos trataron de abordar desde diversas perspectivas ideológicas y con distintos objetivos. La interpretación de la política de estos magistrados que, de un modo u otro, se enfrentaron o trataron de utilizar la cuestión itálica, ha quedado por tanto condicionada por las lecturas tradicionales que predominaban sobre las relaciones entre Roma y los aliados.

El caso de los tribunos de la plebe resulta, en este aspecto, bastante significativo. Esta magistratura se convirtió, desde Tiberio Graco, en la plataforma ideal para aquellos políticos que quisieron llevar a cabo cualquier tipo de proyecto de reforma, bien de corte popular, bien de carácter conservador. Los poderes de los que gozaban los tribunos les permitían abordar cuestiones que quedaban fuera del alcance de otros magistrados, e incluso imponer su voluntad contra el parecer del Senado, utilizando las asambleas populares. Fueron varios los tribunos que, desde el año 133 a.C. y utilizando las prerrogativas de esta magistratura, abordaron la cuestión de los itálicos, tanto como medida de presión para lograr la aprobación de sus proyectos, como con intención de solucionar la ambigua y problemática situación en la que se encontraban los aliados.

\section{LOS ITÁLICOS EN LA ÉPOCA DE LOS GRACO}

Con el tribunado de Tiberio Sempronio Graco comenzamos a documentar en las fuentes de una manera más clara la situación de conflicto entre Roma y los itálicos. Este tribuno tuvo que afrontar el problema de los aliados durante el proceso de aprobación y desarrollo de su ley agraria, hasta el punto de que esta cuestión pudo ser uno de los desencadenantes de su caída. Lamentablemente, la pérdida de la obra de Tito Livio desde el año 168 a.C. hace que nuestra información hasta el tribunado de Tiberio Graco sea muy escasa, por lo que tratar de establecer los antecedentes de la situación en la que éste llevó a cabo sus intentos de reforma resulta una tarea difícil y controvertida. Sin embargo, el silencio de las fuentes no pude interpretarse como la ausencia de una situación de conflicto: para que la cuestión itálica aparezca en los autores que han llegado hasta nosotros es necesario que, o bien dicho conflicto se plasme en actuaciones militares concretas, o bien sea recogida por algún magistrado romano y llevada ante las asambleas o el Senado (Wulff 1991: 194). Con Tiberio Graco, por tanto, aumentan nuestras posibilidades de analizar la situación de los itálicos respecto a Roma, pero esto no significa que no existiera un conflicto previo.

La ley agraria de Tiberio Graco pretendía la limitación de la tenencia de ager publicus a un máximo de quinientos iugera por persona, con la posibilidad de un aumento dependiendo del número de hijos ${ }^{1}$. Sin entrar en la cuestión de la inspi-

\footnotetext{
1 App. BC 1.9.
} 
ración y los objetivos últimos del tribuno, lo cierto es que esta medida pretendía solucionar, mediante el reparto de parcelas de tierra cultivable, el problema del descenso de propietarios agrícolas reclutables para las legiones. Este proyecto tenía como víctimas inmediatas a aquellos individuos que poseían una cantidad de tierras superior a los quinientos iugera permitidos por la ley. Dentro de este colectivo encontramos, en primer lugar, a la aristocracia romana, que había ido acaparando este tipo de campos a lo largo de décadas hasta convertir el ager publicus en una posesión que, si bien seguía perteneciendo al pueblo de iure, en la práctica era explotada como una posesión privada.

Pero, por otro lado, encontramos otro colectivo perjudicado de una forma directa por la lex Sempronia agraria: las oligarquías itálicas que, del mismo modo que la aristocracia romana, habían creado grandes latifundios bajo su dominio con tierras del ager publicus. Es de suponer, además, que las medidas de Tiberio Graco debieron de afectar a las comunidades itálicas en su conjunto, y no sólo a las oligarquías. Si bien el ciudadano romano sin tierras veía en las leyes gracanas una posibilidad de acceder a la condición de propietario, aunque fuera a costa de que la propia aristocracia romana perdiera parte de sus posesiones, el itálico se enfrentaba a una situación en la que unas tierras que antes eran explotadas por un individuo de su pueblo pasaban a manos romanas, sin que él mismo recibiera beneficio alguno en el reparto (Wulff 1991: 199). En suma, los itálicos perdían una considerable cantidad de tierras, sin obtener como contrapartida ningún tipo de compensación. El agravio se producía tanto en el terreno económico como en el ideológico, pues la ley de Tiberio Graco profundizaba, de una manera indirecta, en la situación de subordinación de los itálicos respecto a los ciudadanos romanos.

Otros autores, sin embargo, han considerado que los itálicos sí podrían haber estado incluidos en los repartos de tierras en una primera versión de la ley (Bernstein 1978: 149). Tiberio Graco, ante la más que posible pérdida de popularidad entre la plebe que podía conllevar la inclusión de los aliados en su ley agraria, limitó sus beneficios a los ciudadanos romanos en la versión presentada finalmente a la asamblea. De cualquier modo, parece seguro que los itálicos no podían esperar ganancia alguna de la aplicación de la lex Sempronia.

En el año 133 a.C., por tanto, las exigencias de la nueva ley agraria de Tiberio Graco vienen a sumarse a una situación de por sí vienen desfavorable para las comunidades itálicas. Los repartos de tierras pretendían suplir las bajas en el número total de propietarios causadas por las numerosas guerras exteriores, especialmente largas y costosas en vidas humanas en la segunda mitad del siglo II a.C. Pero las consecuencias de esta sangría constante de ciudadanos no sólo las sufría Roma, sino que también los itálicos, como colectivo que aportaba un alto porcentaje a los efectivos totales de las legiones, habían visto en las últimas décadas cómo el número de varones adultos iba descendiendo en sus comunidades a medida que se prolongaban los conflictos. A esta pérdida continuada de hombres, con una más que dudosa posibilidad de participación en el botín de guerra como única compensación, se añadió el impacto de los repartos de tierras promovidos por Tiberio Graco. Resulta perfectamente comprensible, por tanto, que el descon- 
tento de los itálicos comience en estas fechas a ser un factor a tener en cuenta en la política de la Urbe y, como tal, a aparecer reflejado en nuestras fuentes.

Parece ser que, tras la violenta muerte del tribuno, la actividad de la comisión encargada de aplicar la ley agraria se incrementó en las tierras de los itálicos, ante lo cual los aliados recurrieron a la carismática figura de Escipión Emiliano para que defendiera sus intereses en Roma². Resulta difícil sostener que sea sólo en este momento, tras la muerte de Tiberio, cuando la ley agraria comenzó a afectar a las tierras de los itálicos. Una vez más, el hecho de que nuestras fuentes no informen de ello con anterioridad puede indicar, simplemente, que los aliados no habían encontrado previamente ningún político en la Urbe dispuesto a defender sus intereses. Otras hipótesis han apuntado hacia la existencia de una nueva comisión agraria mucho más hostil hacia los itálicos (Badian 1972: 701). Resulta, por otro lado, bastante lógico que los encargados de llevar a cabo los repartos de tierras se centraran, tras el asesinato del promotor de la ley, en el ager publicus en manos de las oligarquías itálicas en lugar de profundizar en las confiscaciones de los campos controlados por la propia aristocracia romana. La tensión en la ciudad por los repartos había llevado en el año 133 a.C. hasta el extremo de la muerte violenta de un tribuno de la plebe, figura sagrada e intocable, por lo que, tratando de mitigar los enfrentamientos entre los partidarios de la reforma agraria y los terratenientes romanos, la comisión habría preferido continuar su labor con las tierras de los itálicos, sin apenas capacidad política para ocasionar problemas a los gracanos en Roma.

Según el testimonio de Apiano $^{3}$, la comisión agraria habría comenzado en estos momentos a admitir denuncias sobre aquellos terratenientes que violaran la ley de tenencia máxima de tierras públicas. Las dificultades que encontró el colegio de magistrados para aplicar la ley debieron de ser más que considerables si tenemos en cuenta el problemático acceso a la información exacta acerca de qué parcelas constituían o no ager publicus y qué individuos escondían parte de sus posesiones para evitar las confiscaciones. Algunos autores, basándose en hallazgos epigráficos, han llegado a la conclusión de que la comisión agraria habría comenzado su labor por zonas concretas, lo cual delimitaría el terreno a investigar y haría más difícil para los infractores un posible ocultamiento de tierras (Nagle 1970: 390-94). Dado que los aliados no tenían que declarar sus posesiones ante los censores, la labor de la comisión habría resultado en sus tierras especialmente difícil, por lo que la vía de las denuncias creaba una posibilidad más rápida de descubrir a los infractores. Es muy posible que las denuncias tuvieran como incentivo algún tipo de recompensa para el delator (Wulff 1991: 205).

¿Qué intereses pudieron llevar a Escipión Emiliano a asumir la defensa de los itálicos frente a la comisión agraria? En primer lugar, entre el grupo de los gracanos se encontraban algunos de sus más acérrimos adversarios, como el propio Apio Claudio, por lo que Escipión pudo ver en el descontento de los itálicos un medio para oponerse a ellos. Por otro lado, la situación política y militar en Roma había

2 Cic. Mil. 3.8; Rep. 1.31, 3.41; App. BC 1.19.

3 App. BC 1.18. 
cambiado sustancialmente desde el tribunado de Tiberio Graco. La guerra en Numancia se había concluido con éxito gracias al propio Escipión, y las revueltas de Aristónico en Asia y de los esclavos en Sicilia habían sido sofocadas. Todos los factores militares exteriores que habían dado sentido a la ley agraria habían desaparecido, por lo que el general habría visto en el año 129 a.C. una inmejorable oportunidad para terminar con los intentos de reforma y afianzar de una manera definitiva su propio prestigio en la Urbe (Astin 1971).

Escipión consigió que los juicios surgidos a raíz de las denuncias por exceso de posesión de ager publicus no fueran dirimidos por la comisión agraria sino por uno de los cónsules ${ }^{4}$, y arrebató de este modo a los gracanos un poderoso medio de presión contra los infractores de la lex Sempronia. Parece ser que esta medida afectó no sólo a las denuncias contra los possessores itálicos, sino también contra los romanos, por lo que Escipión habría actuado como defensor, no sólo de los aliados, sino también de la aristocracia romana terrateniente, opuesta a Tiberio desde un principio.

Otros especialistas, por el contrario, han señalado la posibilidad de que esta excepción jurídica lograda por el general únicamente se aplicara a los aliados. La principal premisa en la que se apoya esta teoría es que, al ser el Senado, de forma unilateral, quien tomó dicha medida, sin someterla a una ratificación por parte del pueblo, ésta únicamente podría haberse admitido en casos que afectaran a la política exterior, reservados tradicionalmente a la asamblea senatorial (Hardy 1911: 39). Si las relaciones con los itálicos se consideran dentro de este ámbito, el Senado habría podido limitar de este modo la ejecución de la ley tribunicia en su aplicación a las tierras de los aliados.

Hay que observar, por otro lado, que Escipión únicamente arrebató a la comisión agraria la posibilidad de intervenir en los juicios, pero no llegó a atacar el proyecto de ley agraria en su conjunto. Una maniobra política de este tipo habría resultado en exceso arriesgada debido a la popularidad de la que la ley de Tiberio Graco debía de gozar en aquel momento. Aún así, el pueblo romano no admitió que Escipión, el general al que tantas veces había brindado su apoyo en numerosas cuestiones, tomara partido a favor de los itálicos y en contra de los intereses de la plebe romana ${ }^{5}$. Tanto es así que, tras la nunca esclarecida muerte del general, el pueblo no le rindió honores públicos ni exigió una investigación sobre el caso.

Tras la muerte de Escipión, otro tribuno de la plebe, Junio Penno, llevó a cabo una política claramente contraria a los intereses de los itálicos mediante la promulgación de una ley que ordenaba la expulsión de los extranjeros de la ciudad de Roma ${ }^{6}$. Por desgracia, es muy poco lo que sabemos de dicha ley y de su alcance. No obstante, se ha querido ver en la lex lunia Penna tanto un intento de reprimir la llegada de inmigrantes, deseosos de inscribirse como ciudadanos con el objetivo de aprovecharse de los repartos de tierras de la comisión agraria (Bernardi

\footnotetext{
App. BC 1.19; Macr. Sat. 3.14.6-8.

App. BC 1.19.

Cic. Off. 3.47; Brut. 109; Val. Max. 3.4.5.
} 
Ni ciudadanos ni extranjeros: los itálicos en la política de los tribunos de la plebe...

1944-1945: 70), como un intento de limitar la presencia de itálicos en Roma en un momento en el que el candidato a cónsul Fulvio Flaco podía estar incluyendo en su campaña la cuestión de la concesión de la ciudadanía romana a los aliados (Badian 1957: 177).

El siguiente tribuno de la plebe que aparece en las fuentes ligado de alguna forma a la cuestión itálica es Cayo Sempronio Graco, hermano de Tiberio, que continuó con su intento de reforma agraria y social. No entraremos aquí en las motivaciones ni en los objetivos que pudo tener la legislación de Cayo Graco. Señalaremos únicamente que un proyecto de reforma tan ambicioso y de tan largo alcance afectó sin duda de diversas maneras a los aliados itálicos (Wulff 1991: 240). En primer lugar, las medidas llevadas a cabo por este tribuno encaminadas a una mayor protección del ciudadano romano frente a las oligarquías convertían la ciudadanía romana en una situación aún más atractiva para los itálicos.

El proyecto de reforma agraria, la medida legislativa que más había afectado a los itálicos durante el tribunado de Tiberio, no parece que causara en el caso de Cayo Graco malestar alguno entre los aliados ante las confiscaciones y los repartos de tierras. En el caso de Tiberio esta oposición sólo aparece en los textos antiguos gracias a que Escipión se hizo eco de ella, por lo que podría haberse dado el caso de que, ante la pérdida de popularidad del general tras erigirse como defensor de los aliados, ningún político romano hubiera querido continuar su labor. Por otro lado, la carrera política anterior de Cayo Graco le había convertido en una figura cercana a los intereses de los itálicos, con actuaciones como su férrea oposición a la lex lunia Penna en el 126 a.C. ${ }^{7}$ Este hecho, sumado a la adhesión a la causa gracana por parte de otros políticos que se habían mostrado interesados en mejorar la situación de los itálicos durante los años anteriores, pudo generar un clima de esperanza entre los aliados que, ante las nuevas conficaciones de ager publicus, prefirieron aguardar a unas posibles compensaciones por parte del tribuno y su círculo próximo.

Por lo que podemos deducir de las fuentes, Cayo Graco se mostró durante toda su carrera política muy sensible ante la insostenible situación de los aliados itálicos, una actitud que no encontramos en la actuación política de su hermano Tiberio. Sus proyectos de colonización fuera de la Península Itálica pueden interpretarse como un intento de alejar de las tierras de los aliados los problemas derivados de la confiscación de parcelas. Pese a esto, debemos seguir una vez más a Wulff y rechazar la posibilidad de que los propios itálicos hubieran estado incluidos en los proyectos de colonización en África (Wulff 1991: 244). La hipótesis contraria, desarrollada a partir del testimonio de Apiano ${ }^{8}$, no tiene en cuenta los datos ofrecidos por otras fuentes tardías, como Eutropio ${ }^{9}$ y Orosio $^{10}$, que hablan explícitamente de ciudadanos romanos en la colonización, así como ignoran el hecho

\footnotetext{
Cic. Brut. 109.

App. $B C 1.24$.

9 Eutr. 4.12.

10 Oros. 5.12.
} 
de que la inclusión de los aliados hubiera minado considerablemente la popularidad de Cayo Graco ante la plebe romana, algo que no se constata en las fuentes.

Al margen de la cuestión agraria, hubo dos casos más en los que la legislación de Cayo Graco afectó a los itálicos de una manera positiva. En primer lugar, la lex de repetundis permitía acceder a la ciudadanía romana a los aliados que lograran ganar una causa judicial contra magistrados y senadores en casos de abusos económicos. No cabe duda de que esta medida buscaba un control más estricto de la actuación por parte de los magistrados respecto a los aliados, al tiempo que ofrecía a las oligarquías itálicas un camino para acceder a la ciudadanía completa.

Por otro lado, la medida de Cayo Graco que más pudo afectar a los itálicos, y una de las que más controversias ha generado entre los especialistas modernos, es la propuesta sobre la concesión de ciudadanía para los aliados. Las fuentes nos ofrecen diferentes datos acerca del alcance de esta propuesta: desde una concesión de derecho al voto para los itálicos y de ciudadanía completa para los latinos ${ }^{11}$, hasta la extensión de la ciudadanía romana para todos los aliados ${ }^{12}$. No podemos entrar aquí en las distintas interpretaciones que sobre estos testimonios se han elaborado acerca del número de leyes de ciudadanía que Cayo Graco pudo promover o en qué momento de sus tribunados consecutivos llevó a cabo dichas propuestas (Fraccaro 1957: 37). Lo que resulta evidente es que existía un claro deseo por parte de los aliados, tanto de los latinos como de los itálicos, de acceder a la ciudadanía romana completa, y que Cayo Graco entendió la necesidad de satisfacer esta demanda como vía de salida para una situación que comenzaba a resultar peligrosa para la estabilidad política y militar de la propia Roma, tal como la revuelta de Fregella había demostrado en los años anteriores. El itálico era plenamente consciente de su situación de inferioridad respecto al ciudadano romano, y veía en el acceso a la ciudadanía, bien latina, bien optimo iure, una salida para dicho problema. Cayo Graco, por su parte, habría entendido la necesidad de integrar de una manera más efectiva a los aliados itálicos en el cuerpo cívico del Estado, tanto para facilitar sus propios proyectos de reforma agraria, como para, desde una perspectiva más amplia, crear un sistema más sólido y coherente en el cual apoyar el dominio de Roma sobre el Mediterráneo.

El fracaso de la política de Cayo Graco se selló con la muerte del tribuno y sus seguidores a manos de la oligarquía senatorial. Desde este momento, el Senado y los políticos conservadores interpretarán cualquier propuesta de ampliación de la ciudadanía romana a los aliados como un intento de subversión contra el poder de Roma. Los itálicos, por su parte, habían visto cómo en un lapso de diez años habían sido asesinados los dos únicos políticos romanos que se habían mostrado interesados de una manera más o menos activa en la defensa de sus intereses. Escipión Emiliano y Cayo Graco, si bien fueron antagonistas en la política y cada cual actuó siguiendo motivaciones muy distintas, quedan asociados en sus intentos de mejorar la situación de los aliados dentro del sistema político romano.

11 App. BC 1.23; Plut. CG. 5.1, 8.3, 9.3.

12 Vell. 2.6. 


\section{DE LOS GRACO A LIVIO DRUSO}

Tras la muerte de Cayo Graco nuestras fuentes vuelven a guardar silencio acerca de la cuestión de los itálicos. El periodo de aparente calma que se abre hasta la última década del siglo I a.C. no parece roto por nuevas peticiones de ciudadanía o intentos de alzamiento militar contra Roma por parte de los aliados. La ley agraria epigráfica del 111 a.C., así como otras medidas legislativas recogidas por Apiano ${ }^{13}$, muestran cómo la reacción senatorial, si bien respetó las asignaciones de tierras ya realizadas por los Graco, paralizó el proceso de reparto y las confiscaciones.

Aunque Lucio Apuleyo Saturnino, tribuno en el 103 a.C., llevó a cabo un proyecto de reformas que parecía querer continuar el iniciado por Cayo Graco veinte años antes, no recogió entre sus propuestas medida alguna relativa a la integración de los itálicos en el cuerpo político romano. El ejemplo de Cayo, muerto entre otras causas por haber pretendido ampliar la ciudadanía romana a parte de los aliados, debió de pesar tanto en Mario como en los políticos de su círculo, de los cuales Saturnino fue, sin duda, una pieza muy significativa. La amenaza de los pueblos del norte de Europa, así como las circunstancias que envolvieron la guerra de Yugurta, contribuyeron a que el problema de los itálicos quedara, al menos en apariencia, en un segundo plano de la actualidad política romana.

Hay que esperar al año 91 a.C. para que un tribuno de la plebe vuelva a contemplar dentro de sus proyectos políticos la cuestión de los aliados. Marco Livio Druso propondrá, desde una perspectiva conservadora -y alejada, por tanto, de las posturas de Tiberio y Cayo Graco-, la extensión de la ciudadanía romana a todos los itálicos como parte de un complejo programa de reformas. Sin embargo, una vez más, la defensa de los intereses de los aliados, aunque estuviera incluida dentro de un paquete de medidas conducentes a aumentar el poder del Senado, resultó una maniobra condenada al fracaso debido al escaso apoyo que encontró entre la oligarquía romana. Las buenas relaciones del tribuno con algunos miembros de las élites itálicas pronto hicieron recaer sobre él sospechas y acusaciones de búsqueda de un poder personal de tipo tiránico (Bancalari 1987).

Aunque la información que nos ofrecen las fuentes sobre el tribunado de Livio Druso son escasas, podemos observar que es precisamente la cuestión de la ciudadanía para los itálicos lo que fue debilitando su popularidad en Roma. La labor de propaganda en contra del tribuno debió de ser intensa y provenir además de varios grupos contrarios a sus medidas de reforma, tal y como vemos en el dudoso juramento de fidelidad que supuestamente los itálicos hicieron ante Druso ${ }^{14}$.

El asesinato del último defensor de los itálicos demostró a las comunidades aliadas que Roma difícilmente aceptaría una salida política para su situación de subordinación. Todos los magistrados que habían intentado tratar esta cuestión de una manera favorable para los aliados habían acabado muertos, con indepen-

13 App. BC 1.27.

14 Diod. 37.11. 
dencia de su ideología. El malestar que existía en estas comunidades desde tiempo atrás se convirtió entonces en una ausencia total de esperanzas en una solución pacífica. Cuando el procónsul Q. Servilio es asesinado en Ásculo junto con toda la población romana ${ }^{15}$, esta ausencia de esperanza tomó la forma de una férrea determinación: el deseo de obtener la ciudadanía romana dio paso a la lucha por la supresión de la hegemonía de Roma mediante el uso de las armas.

\section{BIBLIOGRAFÍA}

ASTIN, A. E. (1971), Scipio Aemilianus, Oxford.

BADIAN, A. E. (1957), «Caepio and Norbanus», Historia 6, 318-346.

- (1972), «Tiberius Gracchus and the beginning of the Roman revolution», ANRW 1, 668731.

BANCALARI , A. (1987), «Gli intervente degli Italici nella lotta politica romana durante il tribunato di Livio Druso (91 a.C.)», SCO 37, 407-437.

BERNARDI, A. (1944-1945), «La Guerra Sociale e la lotte dei partiti in Roma», Nuova Rivista Storica 28-29, 60-79.

BERNSTEIN, A. H. (1978), Tiberius Sempronius Gracchus. Tradition and Apostasy, Ithaca.

FRACCARO, P. (1957), Opuscula II, Pavia.

HARDY, E. G. (1911), Six Roman Laws, Oxford.

MASCIOLI, F. (1942), «Anti-Roman and pro-Italic sentiment in Italian historiography», Romanic Review 23, 366-384.

MOMMSEN, T. (1888), Römische Geschichte, Berlin.

NAGLE, B. (1970), «The failure of the Roman politic process», Athenaeum 48, 372-394.

WULFF, F. (1991), Romanos e Itálicos en la Baja República, Brussels.

15 App. BC 1.38. 